\title{
Evaluation of Acute Physiology and Chronic Health Evaluation Score II in Acinetobacter baumannii Infection/Colonization and its Antimicrobial Resistance Profile in Kanpur, India
}

\author{
Nidhi Pal and R. Sujatha* \\ Department of Microbiology, Rama Medical College and Research Centre, Kanpur, India \\ *Corresponding author
}

K e y w o r d s
APACHE II Score,
Acinetobacter
baumannii,
Mortality,
Resistance pattern.
Article Info
$\begin{aligned} & \text { Accepted: } \\ & 17 \text { June } 2017 \\ & \text { Available Online: } \\ & 10 \text { July } 2017\end{aligned}$

Keywords

APACHE II Score, Acinetobacter baumanni Mortality, Resistance pattern

Article Info

17 June 2017 Available 0

\section{A B S T R A C T}

Acute Physiology and Chronic Health Evaluation Score II (APACHEII) is one of the wellknown scoring systems that is used to rule out severity of illness and Acinetobacter baumannii causes severe morbidity especially in ICU. The aim of this study is to evaluate APACHE II score in A. baumannii infected or colonized patients and find out the antimicrobial resistant pattern of isolates. In this case control study, clinical details were recorded among patients admitted to ICU. All the clinical samples like tips (endotraceal aspirates, tips, central line tips, and catheters tips), aspirates, blood, urine, fluid and pus were collected. A. baumannii and other isolates (infection or colonizer) were identified by different biochemical tests. All the clinical details were recorded to calculate APACHE II score. Antimicrobial resistance pattern was performed by disk diffusion method and resistant pattern were analysed according to CLSI guideline 2016. Total of 34 A. baumannii was isolated from critically ill patients. Clinically and microbiologically analyzed that $20 \mathrm{~A}$. baumannii caused infections and rest 14 was isolated as a colonizer. Mean APACHE II Score was 23.94 \pm 3.78 . While Mean APACHE II score of control cases

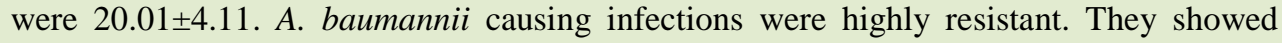
$100 \%$ sensitivity against polymyxins only and $79.4 \%$ resistant for imipenem. But colonizers showed better sensitive for amikacin also. APACHE II Score can be a useful tool to predict $A$. baumannii infections. All the isolated Acinetobacter were highly resistant isolates which restrict the treatment options so it is very essential to treat patients by following all the infection control practices and provide more attention to patients with high APACHE II Score.

\section{Introduction}

Acinetobacter baumannii is highly resistant pathogen and widely distributed in different hospitals as nosocomial pathogen (Ian et al., 2016). This is mostly isolated from intensive care units. In the last decades, the emergence and rapid spread of Multidrug-resistant $A$. baumannii have caused a serious clinical problem worldwide (Peleg et al., 2008). In a developing country concerns about the high costs in the ICU are increasing. Thus illness severity scoring systems have been devised depending on therapeutic, anatomical and physiological basis. Acute Physiology and Chronic Health Evaluation Score II (APACHE II) is one of the well-known scoring systems that are used to rule out severity of illness. This scoring system has been shown to provide objective discrimination between low-risk and high-risk groups of patients (Knaus et al., 1985). 
Severity scoring systems in the intensive care unit have been developed in response to an increased emphasison the evaluation and monitoring of health care services. There are three major purposes of severity-of-illness scoring systems: Scoring systems are used to assess the prognosis of individual patients, to quantify severity of illness and assess ICU performance and compare the quality of care (Gunning et al., 1993).

Currently the APACHE II scoring system is widely used. The APACHE II is measured during the first $24 \mathrm{~h}$ of ICU admission; the maximum score is 71. The APACHE II severity score has shown a good calibration and discriminatory value across a range of disease processes, and remains the most commonly used international severity scoring system worldwide (Knaus et al., 1985; Gunning et al., 1999). The APACHE II index consists of a score that takes account of the patient's age, chronic health condition and physiological variables (internal temperature, heart rate, respiratory rate, oxygenation, arterial $\mathrm{pH}$, sodium, potassium, creatinine, hematocrit, white blood cells and Glasgow coma score).

The aim of the study is to evaluate APACHE II score in Acinetobacter baumannii infected or colonized patients and antimicrobial resistant pattern of isolates.

\section{Materials and Methods}

This case control study 200 cases were observed as a test group and another 200 cases for control group (non Acinetobacter infection) from six different hospitals of Kanpur.

\section{Sample collection}

First ten cases from ICU of each hospital were considered as test cases and observed for only A. baumannii infections while next ten cases were considered as control group and observed for other than Acinetobacter infection.

All clinical details including temperature, arterial pressure, arterial $\mathrm{pH}, \mathrm{Na}+\mathrm{K}+$ values, any underlying diseases and any type of surgeries were recorded within 24 hours of admission in ICU to calculate APACHE Score II (Gunning et al., 1999).

All the clinical samples like endotracheal tips or aspirates, central line tips, blood sample, catheter tips, urine, pus and any fluid samples were collected from test cases and control cases for detection of Acinetobacter and other than Acinetobacter isolates respectively. Acinetobacter baumannii was phenotypically and genotypically identified (Sastry et al., 2016; Bailey and Scott; Howard et al., 2012). Antimicrobial resistant pattern of Acinetobacter baumannii was performed by disk diffusion method and resistant pattern were analyzed according to CLSI guideline 2016 (CLSI, 2013).

\section{Results and Discussion}

In this study 200 each test and control cases were observed. Total 34 Acinetobacter were isolated as infection/colonizer from test cases. The mean APACHE II score of Acinetobacter infected/colonized patients $(23.94 \pm 3.78)$ was more as compared to other than Acinetobacter infected patients $(20.01 \pm 4.11)$ (Table 1). There were 27 patients with A. baumannii colonization/infection had APACHE II score $>21$ and found to be a statistically significant risk factor for Acinetobacter infection/colonizer (Table 2). Mortality rate was $41.17 \%$ (Fig. 1). Among all isolated $A$. baumannii 27 strains were extreme drug resistant A. baumannii (XDR AB). High APACHE II score $(>21)$ were found to have significant $(\mathrm{P}=0.0494)$ and associated with 
mortality (Table 3). Isolated Acinetobacter causing infection or colonization were highly resistant to number of antimicrobial drugs (Fig. 2). Although APACHE II was one of the first systems described, it is still the most widely used. Its calculation is simple, well defined, reproducible, and collected on a routine basis during intensive care service provision. Several studies concerning APACHE II score and increased risk of $A$. baumannii colonization/infection and mortality rate were well documented (Sunenshine et al., 2007; Anunnatsiri et al., 2011; Ntusi et al., 2012). Higher mean of APACHE II score in patients with $A$. baumannii colonization/infection were also documented previously and in accordance with the result of this present study (Emine $e t$ al., 2009).

Table.1 APACHE II score of patients

\begin{tabular}{|l|l|l|l|}
\hline \multirow{2}{*}{ S.N. } & APACHE II Score & $\begin{array}{l}\text { Test group (Acinetobacter } \\
\text { infection/colonization) } n=34\end{array}$ & $\begin{array}{l}\text { Control group (other than } \\
\text { Acinetobacter) } n=75\end{array}$ \\
\cline { 2 - 5 } & Mean $\mathbf{2}$ SD & $\mathbf{2 3 . 9 4 \pm 3 . 7 8}$ & $\mathbf{2 0 . 0 1 \pm \mathbf { 4 . 1 1 }}$ \\
\hline 1. & $>15$ & - & $8(10.6 \%)$ \\
\hline 2. & $16-20$ & $9(2.6 \%)$ & $40(53.3 \%)$ \\
\hline 3. & $21-25$ & $18(52.9 \%)$ & $22(29.3 \%)$ \\
\hline 4. & $>26$ & $7(20.5 \%)$ & $5(6.6 \%)$ \\
\hline
\end{tabular}

Table.2 Patients having APACHE Score >21

\begin{tabular}{|c|c|c|c|}
\hline \multicolumn{5}{|c|}{ APACHE II score (> 21) } \\
\hline No. of isolates & ODD RATIO & $95 \%$ Cl & p-VALUE \\
\hline $25(73.5 \%)$ & 4.9383 & 2.0158 to 12.0977 & 0.0005 \\
\hline
\end{tabular}

Table.3 APACHE Score II (>21) relationship with mortality

\begin{tabular}{|c|l|l|c|c|c|}
\hline & $\begin{array}{l}\text { Test group with >21 } \\
\text { APACHE II score } \\
\text { (Acinetobacter } \\
\text { infection/colonization) }\end{array}$ & $\begin{array}{l}\text { Control group with >21 } \\
\text { APACHE II score } \\
\text { (other } \\
\text { Acinetobacter) }\end{array}$ & ODD RATIO & 95\% Cl & $p$-Value \\
\hline Death & 14 & 26 & 2.6600 & $1.0028-7.0561$ & 0.0494 \\
\hline
\end{tabular}

Fig.1 Mortality Rate among A. baumannii isolated patients

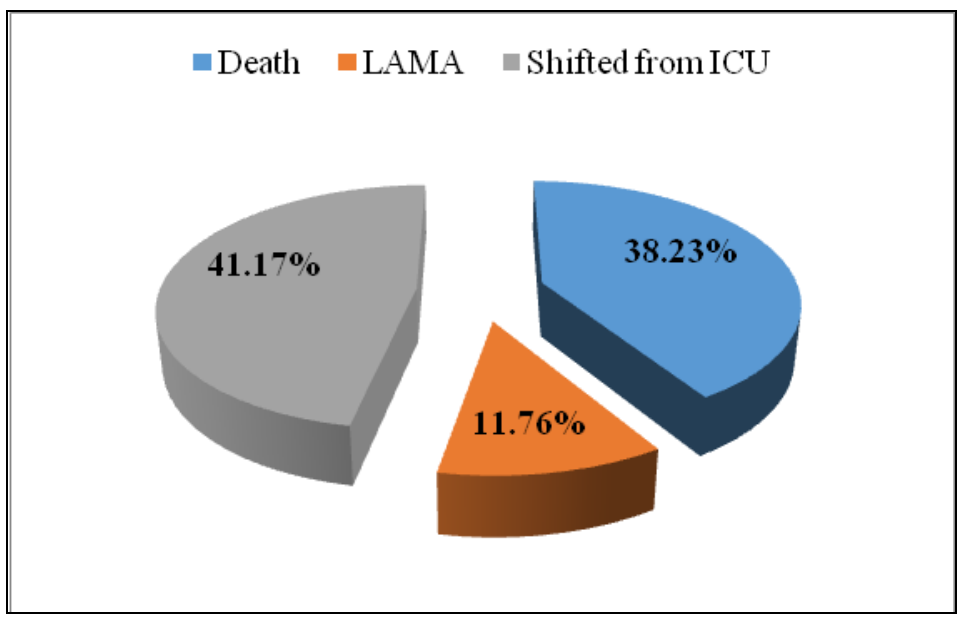


Fig.2 Antibiotic resistance pattern of A. baumannii causing Infection and colonization

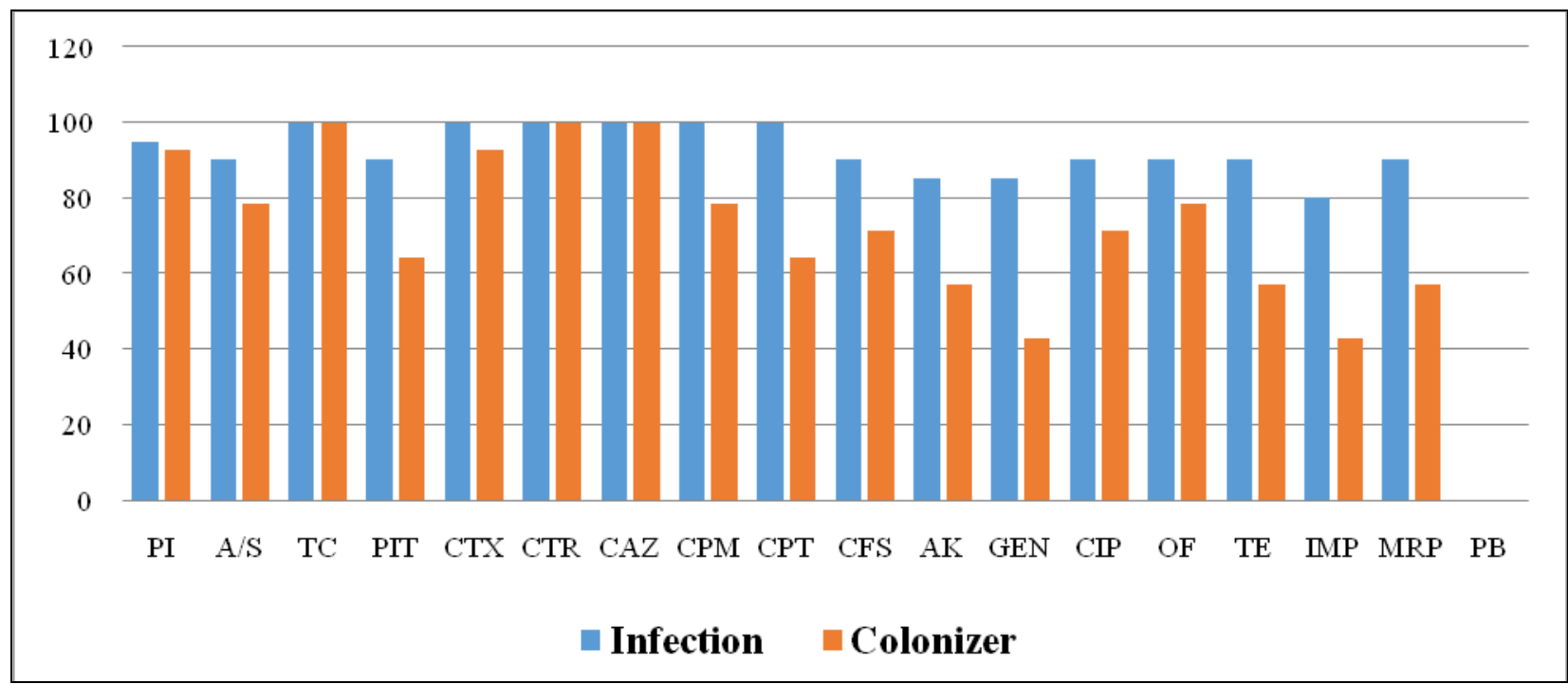

Out of 34 A. baumannii strains, 27 were XDR in this study. In the present study, there were 27 cases with $>21$ APACHE II score, among these from 9 cases were XDR AB. The result of odd ratio may depict risk of $A$. baumannii colonization/infection.

Inappropriate treatment directly affects the mortality of the patients (Michalopoulos et $a l ., 2010)$. In this study, rate of mortality was found as $41.17 \%$. In the other studies, mortality rate of the patients due to $A$. baumannii infections was reported between 22-44\% (Cisneros et al., 1996; Asmita et al., 2012).

In the present study it was observed that all the 14 death cases of $A$. baumannii infection/colonizer were with high $(>21)$ APACHE II score. Its association with mortality was checked with univariate analysis and $>21$ APACHE score $(p=0.0494)$ were found to have significant association with mortality. Lee et al., has recorded that mortality due to $A$. baumannii can be decreased with a proper antimicrobial treatment (Lee et al., 2012). Isolated Acinetobacter causing infection showed maximum susceptibility to polymyxins only
(Vithal et al., 2015). Among isolated strains were $80 \%$ resistant to imipenem and $90 \%$, $100 \%, 85 \%$, and $90 \%$ were resistant to piperacillin-tazobactam, $\quad 3^{\text {rd }}$ generation cephalosporins, amikacin and ciprofloxacin respectively which was contrast to other studies (Ntusi et al., 2012; Emine et al., 2009). While colonizer Acinetobacter show better sensitivity to piperacilline with tazobactum and amikacin (Ismail et al., 2013).

The issue of increased resistance to antibiotics poses difficulty in treatment of $A$. baumannii infections which in turn increases the rate of mortality and cost. In order to prevent development of resistance, antibiotics must be used in an appropriate way in accompanied with proper guidance. If ICU admissions could be prioritized based on scoring systems, the use of limited financial, medical and human resources can be optimized and will allow the best usage in the ICU. Such studies are very few in the developing countries.

In conclusion, APACHE II Score can be a useful tool to predict $A$. baumannii infections. All the isolated Acinetobacter were highly resistant isolates which restrict the treatment 
option so it is very essential to treat patients by following all the preventive measures through proper infection prevention \& control practices and provide more attention to patients with high APACHE II Score.

\section{References}

Anunnatsiri, S., and P. Tonsawa. 2011. Risk factors and clinical outcomes of multidrug-resistant Acinetobacter baumannii bacteremia at a university hospital in Thailand. Southeast Asian J. Trop. Med. Public Health, 42(3): 693703.

Asmita, A., Mehta, V., Anil Kumar, Kumari Indira, K., Suresh, G., Nair, Kavitha, R. Dinesh, Sanjeev K. Singh. Risk factors for mortality in Acinetobacter calcoaceticus-baumannii bacteraemia. Asian Pacific J. Trop. Biomed., S1852S1857.

Bailey and Scott. Diagnostics of Medical Microbiology. Elsevier publication, 13thEdn.

Cisneros, J.M., Reyes, M.J,. Pachon, J., Becerril, B., Caballero, F.J., GarcíaGarmendía, J.L. et al. 1996. Bacteriemia Due to Acinetobacter baumannii: Epidemiology, Clinical Findings, and Prognostic Features. Clin. Infect. Dis., 22: 1026-1032.

CLSI. 2016. Performance standard for Antimicrobial susceptibility testing; twenty third informational supplements. M100-S26, Vol 33 No.1.

Emine, A.L.P., M. Yerer, S. Kocagö, G. Metan, and D. Esel et al. 2009. The risk factors and spread of multidrug-resistant Acinetobacter baumannii in intubated patients in a medical intensive care unit. Turk. J. Med. Sci., 39(5): 761-769.

Emine, A.L.P., M. Yerer, S. Kocagö, G. Metan, and D. Esel, et al. 2009. The risk factors and spread of multidrugresistant Acinetobacter baumannii in intubated patients in a medical intensive care unit. Turk. J. Med. Sci., 39(5): 761769.

Gunning, K., Rowan, K. 1999. ABC of intensive care Outcome data and scoring systems. BMJ, 319: 241-244.

Howard, A., et al. 2012. Acinetobacter baumannii: An emerging opportunistic pathogen. Virulence, 3(3): 243-250.

Ian Huang, et al. 2016. The Role of APACHE-II Score in predicting Acinetobacter baumannii Colonization/Infection and Its Antimicrobial Resistance Pattern in Indonesian Teaching Hospital. American J. Infect. Dis. Microbiol., 4(3): 56-60.

Ismail Necati Hakyemez, Abdulkadir Kucukbayrak, Tekin Tas, Aslihan Burcu Yikilgan, Akcan Akkaya, Aliye Yasayacak and Hayrettin Akdeniz. 2013. Nosocomial Acinetobacter baumannii Infections and Changing Antibiotic Resistance. Pak. J. Med. Sci. 29(5): 1245-1248.

Jung, J.Y., M.S. Park, S.E. Kim, B.H. Park, J. Y. Son, and E.Y. Kim et al. 2012. Risk factors for multi-drug resistant Acinetobacter baumannii bacteremia in patients with colonization in the intensive care unit. BMC Infect. Dis., 10: 228.

Knaus, W.A., Draper, E.A., Wagner, D.P., Zimmerman, J.E. 1985. APACHE II: a severity of disease classification system. Crit. Care Med., 13(10): 818-29.

Lee, Y.T., Kuo, S.C., Yang, S.P., Lin, Y.T., Tseng, F.C., Chen, T.L., et al. 2012. Impact of appropriate antimicrobial therapy on mortality associated with Acinetobacter baumannii bacteremia: relation to severity of infection. Clin. Infect. Dis., 55(2): 209-215.

Michalopoulos, A., Falagas, M.E. 2010. Treatment of Acinetobacter infections. 
Expert Opin. Pharmacother., 11(5): 779-788.

Ntusi, Badri, M., Khalfey, H., Whitelaw, A., and Oliver, S., et al. 2012. ICUassociated Acinetobacter baumannii colonization/Infection in a high HIV prevalence resource-poor setting. PLoS ONE, 7(12): e52452.

Ntusi, N.B.A., M. Badri, H. Khalfey, A. Whitelaw, and S. Oliver, et al. 2012. ICU-associated Acinetobacter baumannii colonization/Infection in a high HIV prevalence resource-poor setting. PLoS ONE, 7(12): e52452.

Peleg, A.Y., Seifert, H., et al. 2008. Acinetobacter baumannii: emergence of a successful pathogen. Clin. Microbiol. Rev., 21(3): 538-82.

Sastry, A.S., et al. 2016. Essentials of Medical microbiology. First Edition. Jaypee brothers medical Publishers.

Sunenshine, R.H., et al. 2007. Multidrugresistant Acinetobacter infection mortality rate and length of hospitalization. Emerg. Infect. Dis., 13(1): 97-103.

Vithal Narayan Dhadke, Shubhangi Dhadke and Vrushali Bhoite. 2015. To Study Ventilator Associated Pneumonia Incidence, Risk Factors and Outcome in Patients on Mechanical Ventilation in Medical Intensive Care Unit. Int. J. Curr. Res. Aca. Rev., 3(10): 35-53.

\section{How to cite this article:}

Nidhi Pal and Sujatha, R. 2017. Evaluation of Acute Physiology and Chronic Health Evaluation Score II in Acinetobacter baumannii Infection/Colonization and its Antimicrobial Resistance Profile in Kanpur, India. Int.J.Curr.Microbiol.App.Sci. 6(7): 1056-1061. doi: https://doi.org/10.20546/ijcmas.2017.607.127 